Falco Edner*, Michael Tornow und Ulrike Steinmann

\title{
Akustische Zeitumkehrfokussierung in Wasser mittels FPGA-basierter Plattform
}

\author{
Acoustic time reversal focusing in water using FPGA based platform
}

\section{DOI 10.1515/teme-2020-0034}

Zusammenfassung: In diesem Beitrag wird das Fokussierverhalten mittels akustischer Zeitumkehr in Wasser diskutiert. Als Grundlage zur Generierung einer hochaufgelösten örtlichen und zeitlichen Fokussierung dient eine eigenentwickelte FPGA-basierte Plattform (auf einem ZynqMP Ultrascale+). Diese ermöglicht eine synchrone Anregung von bis zu 32 Wandlern bei einer Abtastrate von $4,8 \mathrm{MSa} / \mathrm{s}$ und 8 bit Amplitudenauflösung. Zudem wird die gewählte Filter- und Verstärkertechnik vorgestellt und die FPGA-Plattform mit einem kommerziellen analogen System verglichen. Die Messung des Schalldruckfeldes innerhalb der Flüssigkeit erfolgt über ein Nadelhydrophon, das an ein 3D-Schallfeldscanner-System angekoppelt ist. Die im Kalibrierschritt der Methodik erzeugten analogen zeitumgekehrten Impulsantworten werden über eine frequenz- und aufösungsabhängige Pulsweitenmodulation (PWM) für die darauffolgende synchrone FPGAAnsteuerung umgewandelt. Der jeweilige Fokuspunkt wird hinsichtlich seiner Amplitude, räumlichen Auslenkung sowie dem Kontrastverhältnis des Fokuspunktes gegenüber dessen akustisch nicht adressierten Umgebung bewertet. Dabei wird die Fokusqualität bezüglich einer unterschiedlichen Wandleranzahl und an verschiedenen Zielpositionen untersucht.

Schlüsselwörter: Akustische Zeitumkehrfokussierung, FPGA, Synchrone Multi-Kanal-PWM.

Abstract: This paper discusses the focusing behavior by means of acoustic time reversal in water. A self-developed FPGA-based platform (on a ZynqMP Ultrascale+) serves as a basis for generating a high-resolution local and temporal focusing. This platform allows synchronous excitation of up to 32 transducers at a sampling rate of $4.8 \mathrm{MSa} / \mathrm{s}$

\footnotetext{
*Korrespondenzautor: Falco Edner, Lehrstuhl für Messtechnik, Otto-von-Guericke-Universität Magdeburg, Universitätsplatz 2, 39106 Magdeburg, E-Mail: falco.edner@ovgu.de

Michael Tornow, Ulrike Steinmann, Lehrstuhl für Messtechnik, Otto-von-Guericke-Universität Magdeburg, Universitätsplatz 2, 39106 Magdeburg, E-Mail: michael.tornow@ovgu.de, ulrike.steinmann@ovgu.de
}

and 8 bit amplitude resolution. Furthermore, the chosen filter and amplifier technology is presented and the FPGA platform is compared to a commercial analog system. The measurement of the sound pressure field within the liquid is performed by a needle hydrophone coupled to a 3D sound field scanner system. The analog time-reversed impulse responses generated in the calibration step of the methodology are converted via a frequency and resolution dependent pulse width modulation (PWM) for the subsequent synchronous FPGA control. The focus point is evaluated with respect to its amplitude, spatial deflection and the contrast ratio of the focus point to its acoustically unaddressed environment. The focus quality is examined with respect to a different number of transducers and at different target positions.

Keywords: Acoustic time reversal focusing, FPGA, Synchronous multi-channel PWM.

\section{Einleitung}

Die in dem Beitrag genutzte Technik beruht auf dem räumlich-zeitlichen akustischen Zeitumkehrprinzip nach [8-10], um Ultraschallwellen innerhalb einer Flüssigkeit (zum Teil auch durch einen Störkörper hindurch) mit einem Wandlerarray zu fokussieren. Zur Verbesserung des Fokusses bei der akustischen Zeitumkehr kann eine chaotische Kavität genutzt werden. Hierbei wird das Schallfeld durch eine Reihe virtueller Wandler erzeugt, das sich durch Kopplung der chaotischen Kavität an wenige reale Wandler ergibt $[4,6,7]$. Dabei ist aber zu berücksichtigen, dass die maximal eintragbare akustische Energie durch die Anzahl der realen Wandler limitiert ist. Wird die Anwendung auf eine einfache Geometrie beschränkt oder ist es nicht möglich, in dem betreffenden Medium ein Streufeld zu platzieren, dann sollte für die Verbesserung die Anzahl der Schallwandler erhöht werden [5]. Werden eine Vielzahl (realer) Wandler verwendet, so spielt bei der Erzeugung von orts- und zeitveränderlichen lokalen Schalldrücken die synchrone Ausgabe von vielen Kanälen während des Re-Fokussierungsprozesses eine zentrale Rolle. Für die 
Zeitumkehrfokussierung kann durch eine größere Anzahl an synchronen Signalkanälen ein besseres Signal-RauschVerhältnis erreicht werden. Dies konnte bei Versuchen in Festkörpern (Kunststoffplatte) gezeigt werden [2]. Eine weitere Anwendung dieses Prinzips findet sich in [3], wobei dieses Prinzip zur Generierung von räumlich-zeitlichen lokalen Verschiebungen auf einer Platte für eine taktile Multitouch Rückmeldung an einen Anwender genutzt wird.

\section{Theoretische Grundlagen}

Ein Wandler $w$ mit linearem Übertragungsverhalten, angesteuert durch das Spannungssignal $s(t)$ und dessen Faltung mit der Impulsantwort $h(t)$ zwischen dem Punkt $k$ und Wandler $w$, erzeugt an der Position $k$ einen definierten Schalldruck $p(t)$ (Gl. 1).

$$
p_{k}(t)=h_{k w}(t) * s_{w}(t)
$$

Im Frequenzbereich wird aus der Faltung eine Multiplikation (Gl. 2), wobei $H_{k w}$ die Übertragungsfunktion zwischen den Punkt $k$ und Wandler $w$ ist.

$$
P_{k}(\omega)=H_{k w}(\omega) S_{w}(\omega)
$$

Bei simultaner Aktivierung mehrerer Wandler erfolgt eine Addition der Einzelschalldrücke (Voraussetzung lineares System), dass somit:

$$
P_{k}(\omega)=\sum_{w} H_{k w}(\omega) S_{w}(\omega)
$$

Unter Verwendung einer bestimmten Anzahl $W$ an Wandlern und mehrerer Zielpunkte $Z$ innerhalb des Systems folgt die (Gl. 3) in Matrixschreibweise:

$$
\left[\begin{array}{c}
P_{1} \\
P_{2} \\
\vdots \\
P_{Z}
\end{array}\right]=\left[\begin{array}{cccc}
H_{11} & H_{12} & \cdots & H_{1 W} \\
H_{21} & H_{22} & \cdots & H_{2 W} \\
\vdots & \vdots & \ddots & \vdots \\
H_{Z 1} & H_{Z 2} & \cdots & H_{Z W}
\end{array}\right]\left[\begin{array}{c}
S_{1} \\
S_{2} \\
\vdots \\
S_{W}
\end{array}\right]
$$

oder in komprimierter Form:

$$
\boldsymbol{P}(\omega)=\boldsymbol{H}(\omega) \boldsymbol{S}(\omega)
$$

Unter der Verwendung der invertierten Matrix $H^{-1}$ werden die Steuersignale berechnet, die dann zu den gewählten Schalldruckverläufen an den Zielpunkten führen:

$$
\boldsymbol{S}=\boldsymbol{H}^{-1} \boldsymbol{P}_{\boldsymbol{z}}
$$

In den weiteren Betrachtungen wird jeweils nur ein Zielpunkt $z=1$ verwendet, wobei auch mehrere zeitgleiche
Zielpunkte mit jeweils unterschiedlichen Signalen möglich wären.

\subsection{Kalibrierung}

Ein breitbandiges Sinus-Chirp-Signal $v_{w}(t)$ (Frequenzbereich von $0,8 \mathrm{MHz}$ bis $1,1 \mathrm{MHz}$ ) wird an die im Außenbereich des Behälters befindlichen piezoelektrischen Keramiken gegeben (Abb. 1) und zeitgleich wird der jeweilige Schalldruck $p_{k}(t)$ im betreffenden Kalibrierpunkt $k$ mittels Nadelhydrophon gemessen. Dieser Vorgang wird für jeden einzelnen Wandler $w$ und Kalibrierpunkt $k$ wiederholt.

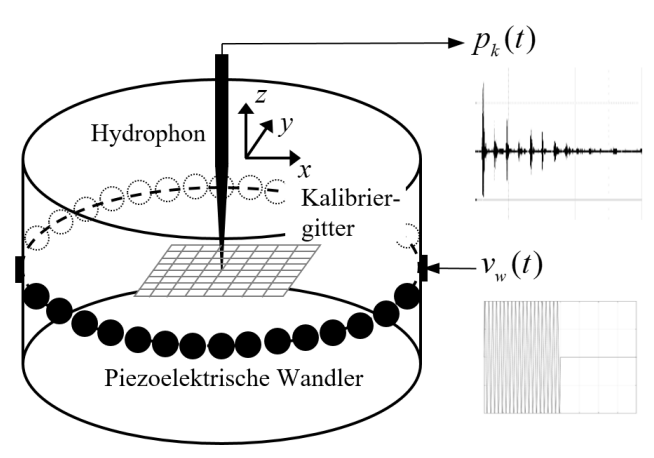

Abb. 1: Testbehälter mit Nadelhydrophon und Kalibriergitter.

Unter Verwendung der beiden Signale kann nach Gl. 7 im Frequenzbereich die Übertragungsfunktion $H_{k w}$ zwischen Wandler $w$ und Kalibrierpunkt $k$ berechnet werden. Dieser Signalvektor dient im Weiteren zur Verrechnung mittels Gl. 6 .

$$
H_{k w}(\omega)=\frac{P_{k}(\omega)}{V_{w}(\omega)}
$$

\subsection{Signalinvertierung}

Die Berechnung des Ansteuersignals $s_{w}(t)$ eines jeden Wandlers $w$ erfolgt über Gl. 6 im Frequenzbereich und einer anschließenden inversen Fouriertransformation mittels Gl. 8. Soll die Re-Fokussierung zu einem bestimmten Zeitpunkt erfolgen, so werden alle $s_{w}(t)$ einheitlich zeitlich verschoben.

$$
s_{w}(t)=F^{-1}(S(\omega))
$$

Das in der experimentellen Verifikation verwendete Zielsignal $p_{z}$ besteht aus einer Periode einer $1 \mathrm{MHz}$ Sinusschwingung mit einer Signallänge von $1 \mu \mathrm{s}$. 


\section{Experimenteller Aufbau}

16 Wandler (bis zu 32 sind im Testaufbau möglich), bestehend aus piezoelektrischen Wandlern der Firma PI Ceramic $\left(10 \times 2 \mathrm{~mm}^{2}\right.$, Material PIC255, Resonanzfrequenz $1 \mathrm{MHz}$ ), werden auf halber Höhe des zylindrischen Behälters (Durchmesser $110 \mathrm{~mm}$, Wanddicke $3 \mathrm{~mm}$, Höhe $200 \mathrm{~mm}$ ) äquidistant als Ring-Array angebracht. Dazu wurden 32 planare Flächen mit einer Tiefe von $1 \mathrm{~mm}$ in den PMMA-Behälter eingefräst, um somit ebene Flächen (Abb. 2 oben) zur besseren Anbringung der Wandler zu schaffen.

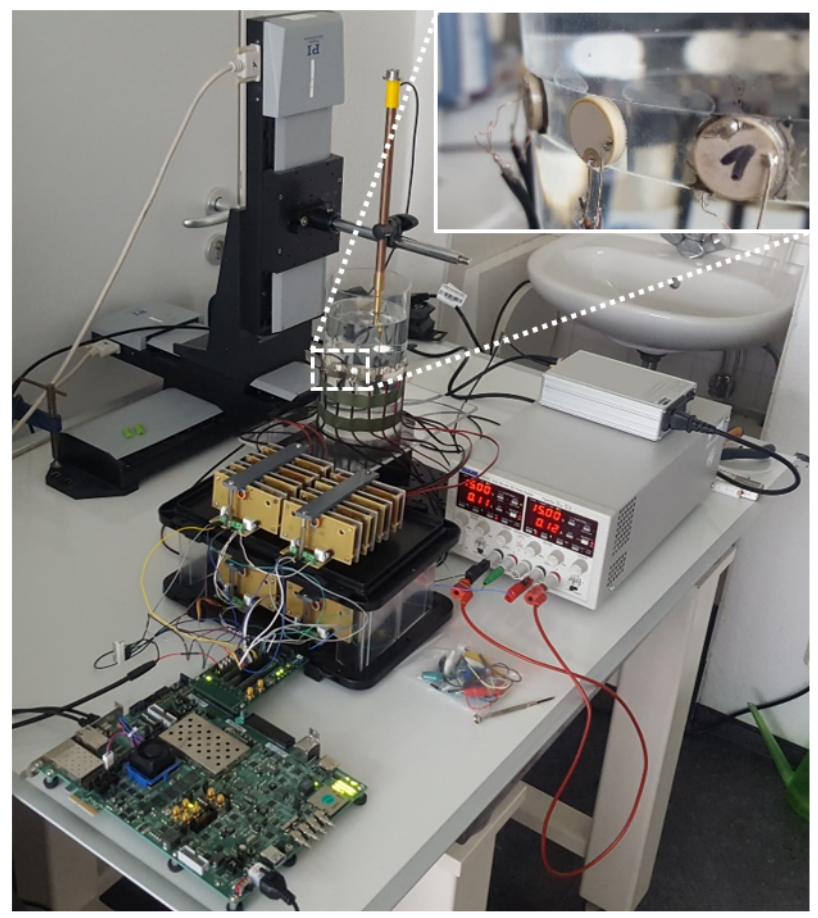

Abb. 2: Experimenteller Aufbau.

Wie weiterhin in Abb. 2 zu sehen ist, besteht der experimentelle Versuchsaufbau aus einem 3D-SchallfeldscannerSystem, das sich aus 3 Hochpräzisions-Lineartischen der Firma Physik Instrumente mit einem Messbereich von $102 \times 102 \times 206 \mathrm{~mm}^{3}$ und einer Schrittweite von 0,4 $\mathrm{\mu m}$ zusammensetzt. An diese Verfahreinheit ist ein Nadelhydrophon (Precision Acoustics NH0500, Frequenzbereich von $0,1 \mathrm{MHz}$ bis $20 \mathrm{MHz}$ ) zur Schalldruckmessung innerhalb der Flüssigkeit angekoppelt. Im Hinblick auf den Durchmesser des aktiven Elementes des Hydrophons von 0,5 mm, wurde die Schrittweite des Messgitters unter Berücksichtigung der zu erwartenden Wellenlänge von ca. 1,5 mm (Flüssigkeit: Wasser, Frequenz: $1 \mathrm{MHz}$ ) auf
$0,5 \mathrm{~mm}$ in $x, y$ und $z$-Richtung festgelegt. Die Aufnahme der Hydrophonsignale erfolgt durch eine Messkarte (National Instruments PXI-5124) mit einer Abtastrate von $200 \mathrm{MSa} / \mathrm{s}$ und einer 14-Bit Auflösung. Die Ausgabe der Signale erfolgt durch die im Folgenden beschriebene FPGA basierte Plattform (ZynqMP Ultrascale+), die bei einer synchronen Ansteuerung von 32 Wandlern eine Abstastrate von 4,8 MSa/s bei einer 8-Bit Aufösung besitzt. Jeder Kanal besitzt zudem einen separaten Verstärker.

\subsection{Synchrone Multi-Kanal-PWM}

Das Aussenden vieler (16 - 32) zeitsynchroner analoger Signale durch Mikrocontroller oder Digitale Signal Prozessoren ist nur mit einem hohen zusätzlichen Hardwareaufwand über eine entsprechend hohe Anzahl an externen D/A-Wandlern realisierbar, da die internen Datenverarbeitungsstrukturen starr sind und die Ausführung der Programme sequentiell erfolgt.

FPGAs hingegen sind für parallele Datenverarbeitung optimiert und in ihrer Struktur sowie ihrer I/O-Funktion programmierbar. Dadurch ist eine Realisierung von vielen analogen Signalkanälen, z.B. über den Einsatz der Pulsweitenmodulation [1] möglich. Hierbei ist für die Ausgabe jeweils nur ein I/O-Pin pro Kanal erforderlich. Darüber hinaus kann durch eine entsprechende Hardware-Struktur im FPGA eine zeitliche Synchronität garantiert werden. Zur Realisierung einer PWM in Hardware ist ein Zähler und ein digitaler Komparator erforderlich. Dies konnte bereits in [2] bei der Ansteuerung der Aktoren einer Kunststoffplatte für Sequenzen im unteren $\mathrm{kHz}$-Bereich gezeigt werden. Zielstellung der FPGA-Plattform war neben der Maximierung der Anzahl der Kanäle und der Länge der Sequenzen auch die Realisierung der Ansteuerung mit bis zu 16-Bit Amplitudenaufösung. Darüber hinaus sollte die Abtastrate flexibel einstellbar sein, um die FPGA-Plattform an unterschiedlichen Versuchsaufbauten einsetzen zu können.

Für den Einsatzbereich Ultraschall in Flüssigkeiten sind aufgrund der höheren Schallgeschwindigkeiten Frequenzen im unteren $\mathrm{MHz}$-Bereich erforderlich. Hier sollte das System noch mindestens 8-Bit Amplitudenaufösung erreichen. Während Zähler und Komparator mit Taktfrequenzen von $1200 \mathrm{MHz}$ realisiert werden müssen, ist die synchrone Datenzuführung der Samples mit diesen Frequenzen nicht realisierbar. Dies ist aber auch nicht erforderlich, da bei 8-Bit Amplituden-Auflösung nur alle 256 Takte neue Sampledaten benötigt werden. Daher wurde das Design mit einem Datentakt von $300 \mathrm{MHz}$ und einem PWM-Takt von $1200 \mathrm{MHz}$ versehen. 
Die Datenübernahme vom Datentakt zum PWM-Takt darf nur vorgenommen werden, wenn die Daten bereits stabil anliegen. Bei der PWM mit $1200 \mathrm{MHz}$, die im Grenzbereich aktueller Arbeitstakte von FPGA liegen, ist ein vollständig synchrones Design erforderlich. Darüber hinaus wurden Register nahe der I/O-Blöcke des FPGAs platziert, welche die PWM-Signale direkt vor der Ausgabe synchron halten. Die Ansteuerung erfolgt über einen im FPGA integierten ARM-A53-Prozessor, der auch die Kommunikation via Ethernet und die Umwandlung der Samples in ein PWM-Signal realisiert. Zur Anpassung unterschiedlicher Abtastraten an die gegenwärtige Konfiguration der Hardware erfolgt bei der PWM-Umwandlung ein Resampling mittels einer linearen Interpolation. Die Sampledaten werden aus dem RAM des Prozessors via Direct Memory Access (DMA) ausgelesen.

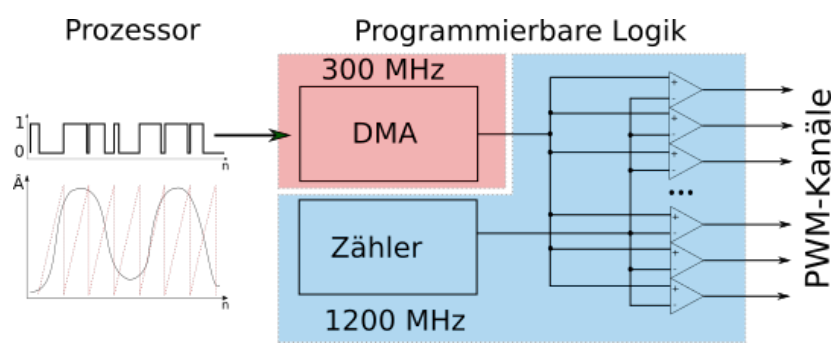

Abb. 3: Synchrone Multi-Kanal-PWM mit $1200 \mathrm{MHz}$.

Die Erzeugung der physischen PWM-Signale erfolgt durch eine Zähler-Digital-Komparator-Einheit, wobei die Periodenlänge und damit die Samplerate flexibel angepasst werden kann. Durch eine höhere Samplerate reduziert sich in gleichem Maße die Amplitudenauflösung. Der 16 Bit-Zähler teilt die Zeit zwischen zwei Samples in 65536 gleiche Teile und wird, wie in Abb. 3 ersichtlich, für den Vergleich der Samplewerte von allen Kanälen verwendet. Bei Nulldurchgang werden alle PWM-Kanäle auf ,High gesetzt. Die Digital-Komparatoren setzen das entsprechende PWM-Signal bei Überschreiten des Samplewertes durch den Zähler auf ,Low'.

Durch den PWM-Takt von $1200 \mathrm{MHz}$ ergibt sich eine Samplerate von $18,3 \mathrm{kHz}$ für eine maximale Amplitudenauflösung von 16 Bit. Die Zähler-Digital-KomparatorEinheit hat 32 Kanäle und wird entsprechend durch $32 \times 16$ Bit (512 Bit/Samplesatz) angesteuert. Der verwendete Custom-DMA-Controller ruft die Samples aus dem RAM ab und kann maximal 2 GB ansprechen. Dadurch ergeben sich bei der Samplerate von $18,3 \mathrm{kHz}$ ca. 230 Sekunden Signallänge. Bei der in diesem Versuch verwendeten Samplerate von 4,68 MHz und einer Amplituden- auflösung von 8 Bit ergibt sich eine maximale Signallänge von 0,89 Sekunden. Die in den Versuchen verwendeten Signale liegen aktuell im unteren $\mu$-Sekundenbereich.

\subsection{Verstärker}

Der Verstärker (Abb. 4) besteht aus einem analogen Operationsverstärker OPA552, an dem ein Bandpassfilter und ein Transformator angebracht sind.

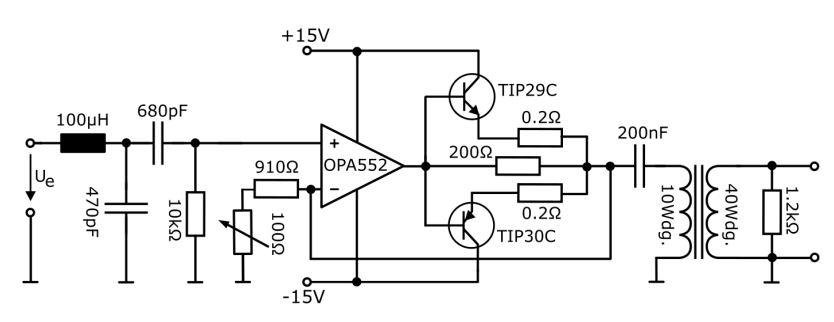

Abb. 4: Verstärkerschaltung mit Filter.

Der Rekonstruktionsfilter besitzt einen Bandpass mit einen Durchlassbereich von $23 \mathrm{kHz}$ bis $734 \mathrm{kHz}$. Hierdurch werden zum einen der Gleichanteil des Signals als auch die Oberwellen, welche durch die PWM entstehen, unterdrückt. Der Transformator im Verhältnis 1 zu 4 verstärkt das Signal weiterhin in dem Bereich der verwendeten hohen Frequenzen, da hier der Operationsverstärker limitiert ist. Der analoge Verstärker wurde gewählt, weil Voruntersuchungen mit einer analogen Ansteuerung erfolgen. Jeder Kanal besitzt zudem einen separaten Verstärker. Das Eingangssignal von 1,8 $\mathrm{V}_{\mathrm{pp}}$ wird auf einen maximalen Ausgangsspannungspegel von $80 \mathrm{~V}_{\mathrm{pp}}$ verstärkt. Wobei die Amplitude durch das Eingangssignal $s_{w}(t)$ vor der PWM-Umrechnung eingestellt werden kann.

\section{Experimentelle Verifikation}

Die eigenentwickelte FPGA-Plattform (Abtastrate von 4,68 MSa/s bei 8 bit Auflösung) soll zunächst gegenüber der kommerziellen analogen Signalgeneratorkarte PXIe5450 der Firma National Instruments (Abtastrate von $10 \mathrm{MSa} / \mathrm{s}$ bei 16 bit Auflösung) verglichen werden. Grundlage hierfür bildet der Re-Fokussierungsprozesses auf den Behältermittelpunkt.

Der Kalibrierprozess erfolgt über das FPGA-System. Die zeitumgekehrten Signale werden durch beide Systeme ausgeben. Durch die verwendete Verstärkertechnik ist dies problemlos möglich. Hierbei ist die Kanalanzahl auf 2 


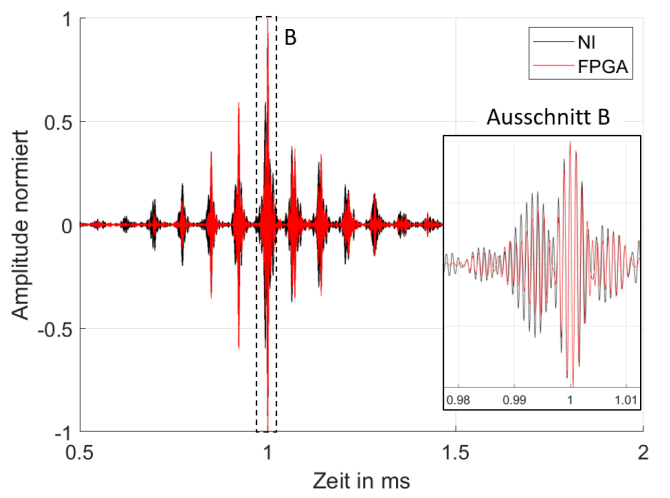

Abb. 5: Gegenüberstellung des zeitlichen Verlaufs des Hydrophonsignals im Behältermittelpunkt bei 2 Wandlern mittels $\mathrm{NI}$ PXle-5450 und FPGA.

durch den NI-Signalgenerator begrenzt. Für die Messung wurden 2 gegenüberliegende Wandler genutzt. Der Signalverlauf beider Systeme bei der Fokussierung stimmt nach Abb. 5 deutlich überein. Hierbei wurden die beiden Signale zur besseren Vergleichbarkeit auf deren jeweiligen maximalen Wert normiert.

Der zeitliche Verlauf des Hydrophonsignals und die Amplitude des Fokuspunktes bei Einsatz von 4, 8 und 16 Wandlern des Arrays soll im nächsten Schritt untersucht werden (Abb. 6). Dabei dient wiederum der Behältermittelpunkt als Zielpunkt, wobei jeweils 2 Wandler sich stets gegenüber stehen und gleichmäßig auf dem Umfang verteilt sind.

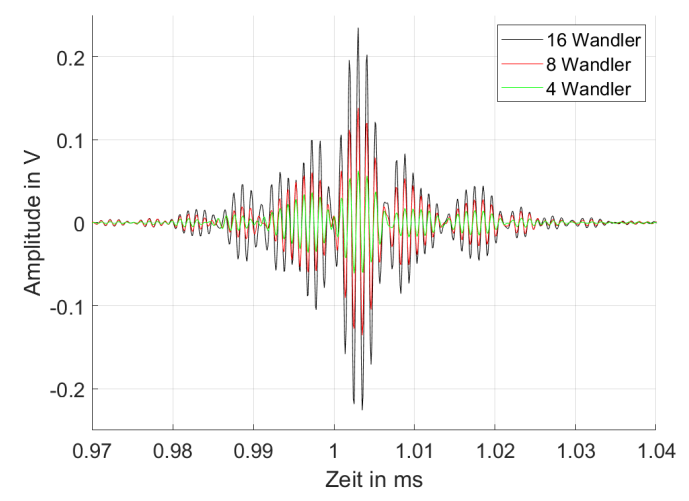

Abb. 6: Zeitlicher Verlauf des Hydrophonsignals im Behältermittelpunkt bei 4, 8 und 16 Wandlern zum Fokuszeitpunkt.

Die Steigerung der Wandleranzahl beeinflusst nicht den zeitlichen Verlauf des Signals, jedoch dessen Amplitude. Bei 4 Wandlern beträgt die maximale Amplitude $63 \mathrm{mV}$, bei 8 Wandlern $138 \mathrm{mV}$ und bei 16 Wandler $235 \mathrm{mV}$. Zu erwarten ist durch das lineare Verhalten des Systems stets eine Verdopplung der Amplitude, was annähernd in den Messwerten erkennbar ist.

Die laterale Verteilung am Fokuspunkt soll nachfolgend als Funktion der Wandleranzahl untersucht werden. Abb. 7 zeigt dazu die in Form der Hydrophonspannung dargestellte Schalldruckverteilung bei Verwendung von 4, 8 und 16 Wandlern.
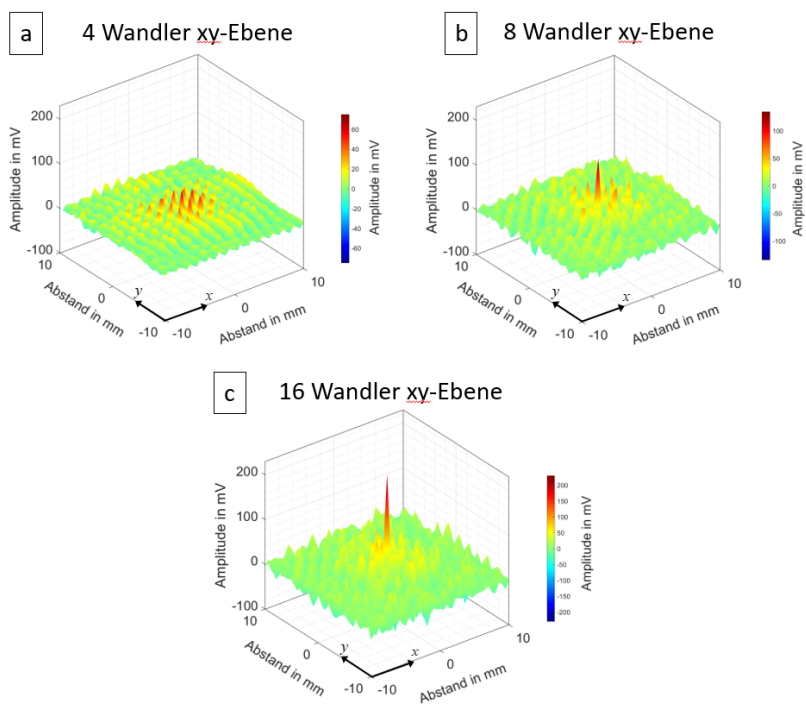

Abb. 7: Amplitudenverteilung des Hydrophonsignals zum Fokuszeitpunkt bei verschiedener Wandleranzahl.

Die $x y$-Ebene der Abb. 7 bezieht sich auf die Wandlerebene (Draufsicht auf den Zylinder). Dabei wurde das Messgitter von $10 \mathrm{~mm}$ x $10 \mathrm{~mm}$ innerhalb der Ebene in $0,5 \mathrm{~mm}$ Teilabschnitte aufgeteilt und es ist eine mit der Wandleranzahl steigende Amplitude im Fokuspunkt erkennbar (vgl. auch Abb. 6). Der Flächenscan zeigt weiterhin, dass sich das Kontrastverhältnis (SNR) zwischen Fokuspunkt und umliegenden Bereich erhöht. Die laterale Amplitudenverteilung des Fokuspunktes von ca. $1 \mathrm{~mm}$ Durchmesser (ausreichendes SNR ab etwa 8 Wandlern) ändert sich bei zunehmender Wandleranzahl nicht. Zu erwarten ist eine Auflösung im Bereich der halben Wellenlänge des Zielsignals der $1 \mathrm{MHz}$ Sinusschwingung im Wasser (ca. 0,75 mm).

Weiterhin wird das Zielsignal $s_{w}(t)$ auf einen außerhalb des Mittelpunktes befindlichen Zielpunkt (Abstand $-5 \mathrm{~mm}$ in Richtung $\mathrm{x}$-Achse) gerichtet. Die betreffenden Drauf- und Vorderansichten des Behältermittelpunktes und Außenpunktes bei Nutzung von 16 Wandlern sind in Abb. 8 dargestellt. Die laterale Amplitudenverteilung im Umkreis des betreffenden Fokuspunktes in xy-Ebene ändert sich nicht, jedoch sinkt die Amplitude von $230 \mathrm{mV}$ 

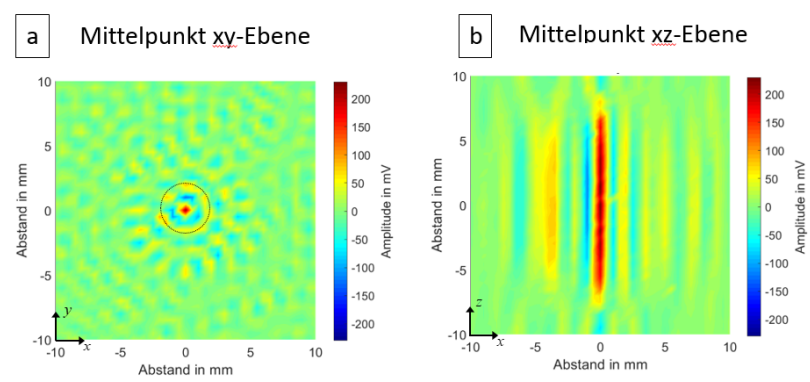

c $x=-5 \mathrm{~mm}$ xy-Ebene
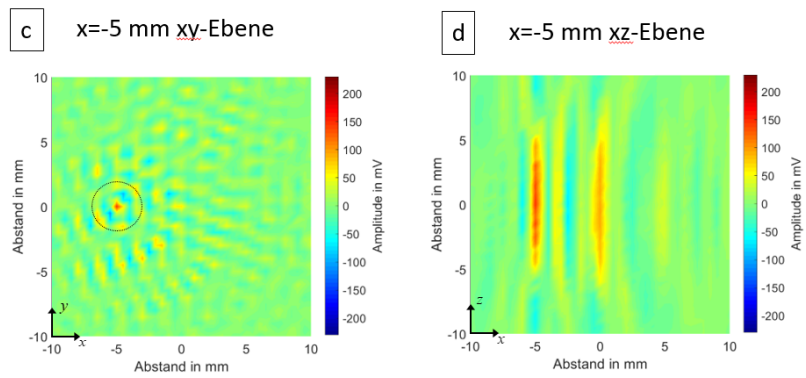

Abb. 8: Amplitudenverteilung des Hydrophonsignals im Behältermittelpunkt und bei einem Zielpunkt-Versatz von $x=-5 \mathrm{~mm}$ (Verwendung von 16 Wandlern).

auf $155 \mathrm{mV}$ ab. Dabei sinkt auch die räumliche Ausdehnung des Fokuspunktes innerhalb der xz-Ebene von ca. $15 \mathrm{~mm}$ im Behältermittelpunkt auf $10 \mathrm{~mm}$ Höhe im auBermittigen Punkt. Es ist damit zu erkennen, dass die Schalldruckamplitude von außermittigen Fokuspositionen unter ansonsten gleichen Bedingungen schwächer als am Behältermittelpunkt ausfällt.

\section{Zusammenfassung Fazit}

In diesem Beitrag wurde die akustische Zeitumkehr unter Ausnutzung einer hohen Anzahl an synchronen Kanälen mittels einer eigenentwickelten FPGA-Plattform untersucht. Der Re-Fokussierungsprozess wird mit einem analogen Signalgenerator verglichen, wobei sich eine hohe Übereinstimmung erkennen lässt. Die Vorteilhaftigkeit der FPGA-Plattform zeigt sich insbesondere bei der Verwendung von mehr als 8 Wandlern, da hier marktverfügbare Standardsysteme hinsichtlich einer Intensivierung der Fokusamplitude gegenüber der akustisch nicht adressierten Umgebung an Grenzen gelangen. Zudem können durch die beschriebene Technik kalibrierte Zielpunkte orts- und zeitaufgelöst angesprochen werden. Eine sehr gute Kopplung zwischen den Wandlern und dem Zielfokus kann im Zentrum des zylinderförmigen Behälters festgestellt werden, während sie außerhalb davon abnimmt.

\section{Literatur}

[1] M. Barr. Pulse width modulation. Embedded Systems Programming, S. 103-104, 2001.

[2] F. Edner, M. Tornow und U. Steinmann. FPGA basierte Plattform zur akustischen Zeitumkehrfokussierung. Fortschritte der Akustik - DAGA 2020: 46. Jahrestagung für Akustik, S. $142-145,2020$.

[3] C. Hudin und S. Panëels. Localisation of vibrotactile stimuli with spatio-temporal inverse filtering. In Haptics: Science, Technology, and Applications, Band 10894 of Lecture Notes in Computer Science, S. 338-350. Springer International Publishing, 2018. 10.1007/978-3-319-93399-3_30.

[4] J. Robin, M. Tanter und M. Pernot. A semi-analytical model of a time reversal cavity for high-amplitude focused ultrasound applications. Physics in medicine and biology, 62(18):7471-7481, 2017. ISSN 1361-6560. 10.1088/1361$6560 /$ aa8211.

[5] Roux und Fink. Time reversal in a waveguide: study of the temporal and spatial focusing. The Journal of the Acoustical Society of America, 107(5 Pt 1):2418-2429, 2000. ISSN 0001-4966. 10.1121/1.428628.

[6] A. Sarvazyan, L. Fillinger und L. Gavrilov. Time-reversal acoustic focusing system as a virtual random phased array. IEEE transactions on ultrasonics, ferroelectrics, and frequency control, 57(4):812-817, 2010. ISSN 0885-3010. 10.1109/TUFFC.2010.1486.

[7] Y. D. Sinelnikov, A. M. Sutin und A. P. Sarvazyan. Time reversal in ultrasound focusing transmitters and receivers. Acoustical Physics, 56(2):183-193, 2010. ISSN 1063-7710. 10.1134/S1063771010020107.

[8] M. Tanter, J.-L. Thomas und M. Fink. Time reversal and the inverse filter. The Journal of the Acoustical Society of America, 108(1):223-234, 2000. 10.1121/1.429459.

[9] M. Tanter, J.-F. Aubry, J. Gerber, J.-L. Thomas und M. Fink. Optimal focusing by spatio-temporal inverse filter. i. basic principles. The Journal of the Acoustical Society of America, 110(1):37-47, 2001. 10.1121/1.1377051.

[10] F. Vignon, J.-F. Aubry, A. Saez, M. Tanter, D. Cassereau, G. Montaldo und M. Fink. The stokes relations linking time reversal and the inverse filter. The Journal of the Acoustical Society of America, 119(3):1335-1346, 2006. ISSN 00014966. $10.1121 / 1.2161452$. 